\title{
Health Related Quality of Life in Functional Gastrointestinal Disorders in Asia
}

\author{
Myung-Gyu Choi ${ }^{1 *}$ and Hye-Kyung Jung ${ }^{2}$ \\ ${ }^{1}$ Department of Internal Medicine, College of Medicine, The Catholic University of Korea, Seoul, Korea, ${ }^{2}$ Department of Internal Medicine, Ewha \\ Womans University School of Medicine, Seoul, Korea
}

The importance of health-related quality of life (HRQOL) continues to grow, as clinicians and clinical researchers have recognized the impact of the functional gastrointestinal disorders. Limited information is available on the performance of $\mathrm{HRQOL}$ questionnaires in Asia. Furthermore, the effect across different cultural settings of functional gastrointestinal disorders on HRQOL has been little studied in Eastern countries. We summarized recent studies on HRQOL in Korean patients with functional gastrointestinal disorders as well as other Asian literatures. Functional gastrointestinal disorders-related symptoms had a great effect on the HRQOL of Korean patients. These results and their considerable prevalence in Korea indicate that functional gastrointestinal disorders have a substantial social impact in this country.

(J Neurogastroenterol Motil 2011;17:245-251)

\section{Key Words}

Dyspepsia; Irritable bowel syndrome; Quality of life

\section{Introduction}

Functional gastrointestinal disorders (FGIDs) are chronic conditions that are considered to have no structural or biochemical abnormalities that account for the symptoms. ${ }^{1}$ FGIDs are not life threatening conditions, however, they significantly could impair the quality of life (QOL). FGIDs are important to public health because they are remarkably common, can hinder daily activities and induce a major social and economic burden. Thus, the impact of FGIDs on population health is probably huge. Despite its potential health relevance, the impact of FGIDs has received relatively little attention compared to other life threatening diseases in Asia. Furthermore, the effect across different cultural settings of FGIDs on health-related quality of life (HRQOL) has been little studied in Asian countries. The majority of published studies on FGIDs are ethnocentric and mostly reported from Western, Caucasian populations. Recently, more attention was given to the health economic implication of FGIDs in Asia along with the progress of economy. Here, we summarized recent studies on HRQOL in Asian patients with FGIDs.

Received: May 25, 2011 Revised: June 10, 2011 Accepted: June 12, 2011

(C) This is an Open Access article distributed under the terms of the Creative Commons Attribution Non-Commercial License (http://creativecommons. org/licenses/by-nc/3.0) which permits unrestricted non-commercial use, distribution, and reproduction in any medium, provided the original work is properly cited.

*Correspondence: Myung-Gyu Choi, MD

Division of Gastroenterology, Department of Internal Medicine, The Catholic University, Seoul St. Mary's Hospital, 505 Banpodong, Seocho-gu, Seoul 137-701, Korea

Tel: +82-2-2258-6017, Fax: +82-2-2258-2089, E-mail: choim@catholic.ac.kr

Financial support: This work was supported by a grant from National Evidence-based Healthcare Collaborating Agency of Korea (NA-18). Conflicts of interest: None. 


\section{Definition and Assessment of Health-Related Quality of Life in Asian Literature}

World Health Organization defined health as condition that has the absence of disease and infirmity but also has the presence of physical, mental, and social well-being. ${ }^{2}$ QOL can be defined as the functional effect of an illness and its consequent therapy upon a patient. It describes health status from the patients' perspective. In this context, when we are managing patients, we must care not only about objective findings but also about patient's perception. HRQOL is made up of subject perceptions and objective health status which comprised of physical, psychological and social domain. The assessment of HRQOL continues to grow in importance, as clinicians and clinical researchers have recognized the impact of the FGIDs.

Both generic and disease-specific QOL instruments are to assess HRQOL. Generic instruments use generalized questions that are not specific to any particular diseases. An example of commonly used generic questionnaires in Asian studies is the Short Form 36 Health Survey (SF-36). It has 36 scaled items about health. Items are clustered in sub-scores (domain), such as "vitality" and "mental health". Lower scores indicate poorer QOL, with a score of 100 indicating perfect health status. There are published normative data for the general population of several Asian countries, ${ }^{3-5}$ facilitating comparisons between the subjects with disease and healthy controls. Generic instruments are used in general populations to assess a variety of health status, conditions, and diseases. They also permit comparison among diseases and may even detect unanticipated effects. However, they are time consuming and complicated with multiple questions. Generic instruments are not specific tools to assess any particular disease status or susceptible population of patients. Thus, they are insensitive to change with time or treatment. Disease-specific questionnaires are designed to detect HRQOL changes that may not be targeted in generic instruments, but are important manifestations of a certain disorder. Disease-specific instruments may not be widely applied to other diseases as these symptoms may not appear in other disorders. The advantage over the generic instruments is that they reflect problems most important to a specific population. They can detect smaller and more specifically relevant changes with time or treatment in health status, which may be overlooked by generic instruments. Disease-specific instruments are also too complex to administer and score. They are not capable of discriminating among different medical conditions.
Examples of commonly used disease-specific questionnaires in FGIDS in Asian studies are the IBS-QOL and the Nepean Dyspepsia Index (NDI). ${ }^{6-8}$ The IBS-QOL was developed by Patrick et al, ${ }^{9}$ which was comprised of 34 irritable bowel syndrome (IBS) specific QOL items with 8 Domains: dysphoria, interference with activity, body image, health worry, food avoidance, social relations, sexual relations and relationships. Each items was rated using a 5 -point Likert response scale. Those scales are added up and transformed to 0-100 scale, indicating 0 as the poorest QOL and 100 as the maximum QOL. A patient responding "not at all" for all 34 items would score 100 in IBS-QOL. If responded as "slightly", for all items, the overall IBS-QOL score would be 80. NDI is a dyspepsia-specific QOL instrument developed by Talley et al. ${ }^{10}$ This is a measuring instrument of symptom status and QOL in functional dyspepsia. A 15 -item symptom checklist measures the frequency, severity, and bothersomeness of each upper gastrointestinal symptom on Likert scales. A total score for each symptom is created by adding 3 scales. A total dyspepsia HRQOL score is derived from the 25 -item NDI, which is comprised of 5 sub-domains; interference, knowledge/control, eat/drink, sleep disturbance and work/study. ${ }^{11,12}$

Humans interpret health events in a manner that is partly dependent on their social and cultural background. Thus, sociocultural validation of a questionnaire is needed to see its relevance to sociocultural influence. In most Asian studies, translation of the original questionnaires was done appropriately according to the accepted linguistic validation guidelines, including forward translation, backward translation and patient testing before their use. In addition to the linguistic validation, cross-cultural validation was performed in some studies. ${ }^{13-15}$

We performed a systematic review of the literature to compare the HRQOL of patients with FGIDs with either healthy controls or those with other diseases. Full-length published papers from Asia during 1980-2010 were included if (1) patients had FGIDs and (2) HRQOL was measured using validated generic or dyspepsia-specific instruments, but not global assessment alone.

\section{Chronic Gastrointestinal Symptoms and Their Effect on Health-Related Quality of Life in Community}

Only a few studies were conducted on HRQOL in people in the community who did not visit the clinic (Table 1). One study from Korea has conducted a cross-sectional survey of gastro- 


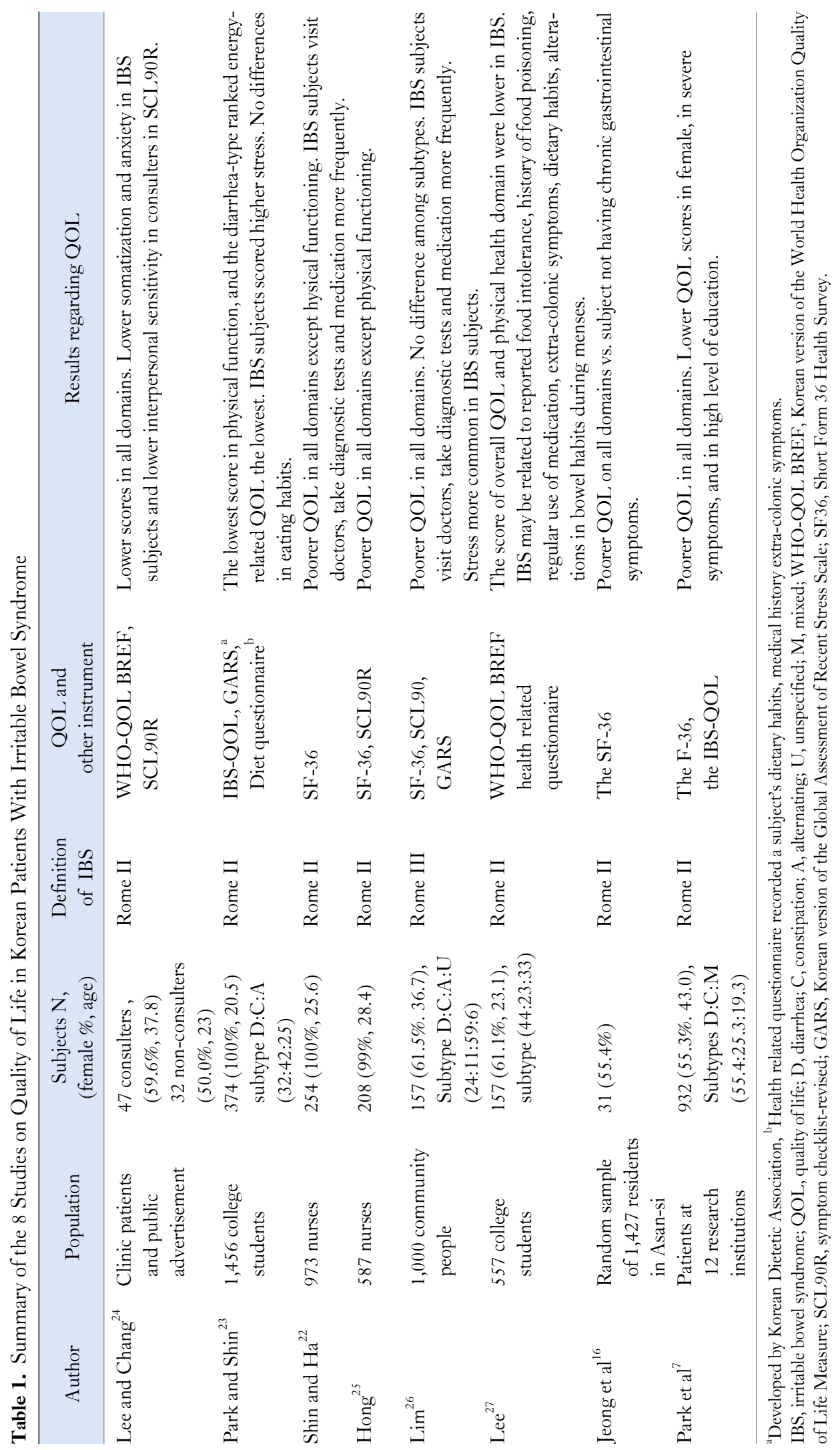




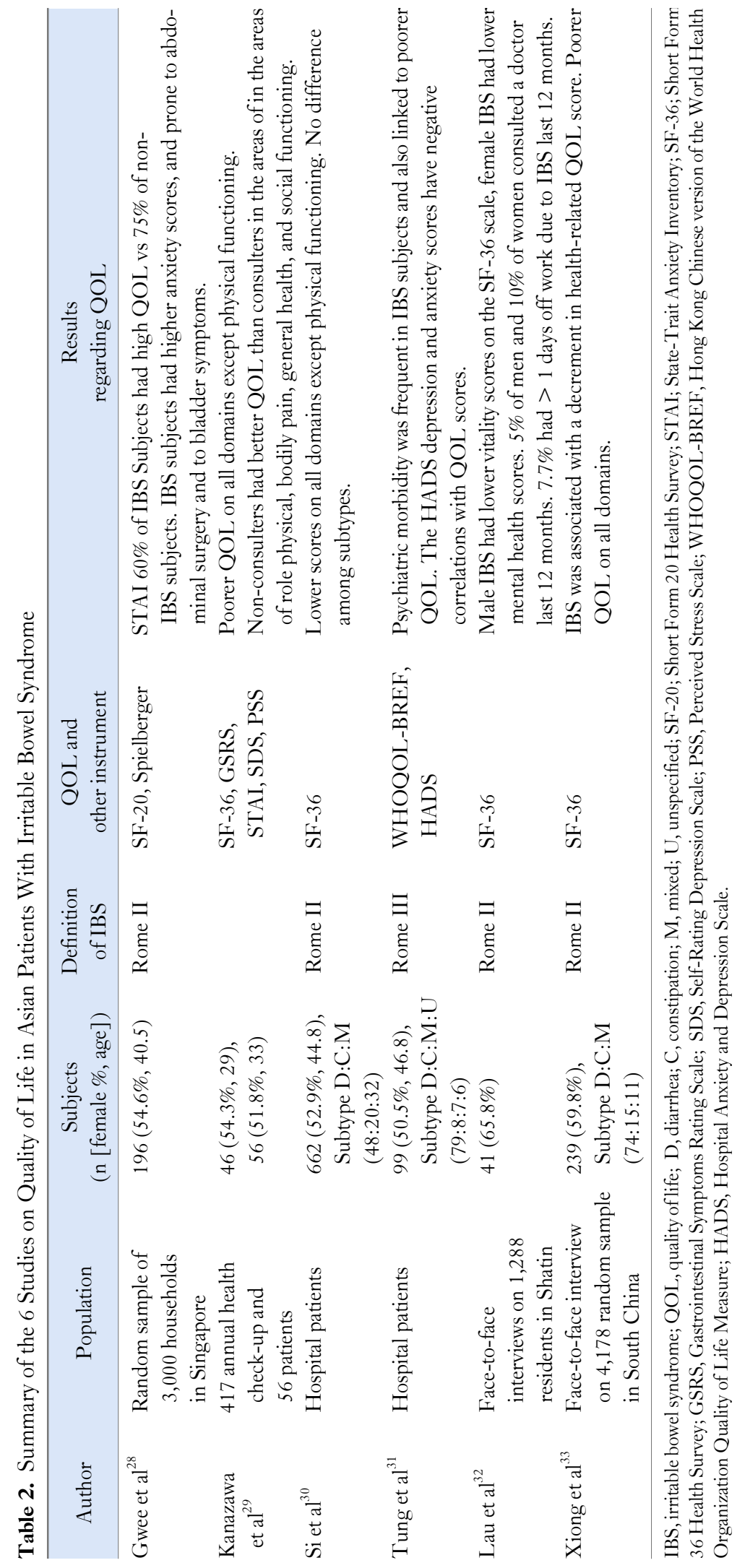

intestinal symptoms and its impact on QOL in the community. ${ }^{16}$ They picked the city of Asan with population of 120,000 as a representative sample of Koreans. The survey included randomly selected 2,024 adult residents. All subjects underwent face-to-face interviews and the response rate was $78.4 \%$. There was a high prevalence rate of chronic gastrointestinal symptoms in the community not seeking medical care. At least 1 chronic gastrointestinal symptom was present in $18.6 \%$ of those surveyed. Dyspepsia was most common and reported in $11.7 \%$. Gastroesophageal reflux disorder defined as weekly heartburn was present in $3.5 \%$, IBS by Rome II criteria in $2.3 \%$, bloating in $4 \%$, and constipation in $2.6 \%$. HRQOL was significantly impaired among the subjects with gastroesophageal reflux disease, dyspepsia and irritable bowel syndrome compared to healthy controls. Both mental and physical scores were similarly reduced in non- consulters with chronic gastrointestinal symptoms in this study. These findings are in direct contrast to other studies which demonstrated a normal range of HRQOL in non-consulters. Discrepancy might be related with strict time criteria of chronicity of this study which was over 3 months per year.

\section{Functional Dyspepsia}

Functional dyspepsia is believed to be common in Asia as well as in Western countries. In Korea, it has been estimated that approximately $12 \%$ of the adult population have dyspepsia, ${ }^{16}$ and that large proportion of sufferers have functional rather than definite organic causes for their symptoms. The management of dyspepsia remains a major issue in clinical practice. Functional dyspepsia is the most common FGID in both primary care and gastroenterology practice in Korea. ${ }^{17}$ The nationwide dyspepsia study in Korea was performed in $2002 .^{18}$ The subjects of this survey included 3,038 patients with dyspepsia seen in both referral and primary care centers in each of the 10 regions in South Korea. All patients underwent endoscopy and completed a dys- 
pepsia questionnaire and the SF-36. Endoscopic examination revealed the organic causes of dyspepsia in $28 \%$ of dyspeptic patients; gastric ulcer in $5.3 \%$, duodenal ulcer in $9.3 \%$, gastric cancer in $1.4 \%$ and reflux esophagitis in $2.6 \%$. Patients with functional dyspepsia by Rome II criteria had lower HRQOL score on all 8 domains of the SF-36 compared to general population. Both mental and physical scores were equally lower than healthy subjects. Patients with functional dyspepsia reported poorer QOL in 4 domains including general health, vitality, role emotional and mental health than the patients with peptic ulcer. Dyspeptic patients who are seen in a referral setting had worse HRQOL scores than primary care patients and non-consulters who were detected in the general population.

Cho et $\mathrm{al}^{6}$ evaluated QOL using NDI. Validation study of Korean version of NDI revealed an excellent test-retest reproducibility and internal consistency which was expressed by Cronbach's alpha, 0.92. Construct was validated by comparison with SF-36, global rating of QOL, and anxiety and intrusiveness. The scores of all subscales were significantly low in dyspeptic patients demonstrating that the NDI is able to discriminate between the 2 groups. The greatest impairment was seen in the knowledge/control domain of all subscales, and the lowest impairment was seen in the sleep disturbance subscore. Responsiveness of the NDI instrument was performed as part of the cross-cultural validation, which took place in 3 centers in an open trial of mosapride. ${ }^{6}$ One hundred and 29 patients with functional dyspepsia by Rome II participated in this study and completed the SF-36 and the NDI at baseline and the NDI after mosapride treatment. All dyspeptic symptoms were significantly decreased after mosapride treatment. Whereas dyspepsia score decreased after medication, NDI score increased after treatment. In other words, patient's symptoms improved as well as their QOL. An increase of NDI score after mosapride treatment, which meant a better QOL, was observed in summary scale as well as in all 5 subscales. The greatest improvement was observed in the knowledge/control domain among 5 subscales which was mostly impaired prior to treatment. This could explain the nature of improvement after drug treatment. The subjects were compared as responders and non-responders to mosapride treatment for evaluating the degree to which the NDI questionnaires and their subscales improved with treatment. All NDI subscales showed 154\%-302\% greater improvement for responders over nonresponders $(P<0.001$ for all subscales). This finding attests to the fact that the NDI is sensitive enough to detect changes with treatment. In this study the NDI was shown to be highly re- sponsive to treatment effects thereby leading to its recommendation to be used in treatment trials. However, further studies are needed in the large placebo controlled trials.

\section{Irritable Bowel Syndrome}

The reported prevalence of IBS in Asian countries varies from 5 to $10 \%$ depending on the study population and diagnostic criteria (Table 2). ${ }^{19,20}$ Asian doctors and medical professionals are beginning to notice the burden of IBS along with increasing prevalence of the organic colonic diseases in Asian countries. A Taiwanese survey assessed the social and sleep quality impact of IBS subjects receiving physical check-up. ${ }^{21} 56 \%$ of IBS subjects visited physicians due to gastrointestinal symptoms and the mean number of visits was 2.3 during the previous year. The absenteeism from work or school was marked in the IBS subjects, whether due to any illness or any particular gastrointestinal symptoms. An almost 2-fold increase in the frequency of sleep disturbance was noted in subjects with IBS symptoms. ${ }^{21}$ Studies regarding QOL in Korean and Asian patients with IBS were summarized in Tables $1^{7,16,22-27}$ and 2. ${ }^{28-33}$ Most studies consistently showed poorer QOL in IBS subjects in both mental and physical summary of the SF 36, while some showed no difference in physical functioning domain. Poorer QOL in mental component can be explained by psychiatric comorbidity in IBS subjects. Even though the social and cultural environment in which study was carried out differ from that in the West, the results are in agreement with those studies conducted in the West. The QOL of IBS patients was significantly impaired in all dimensions of generic HRQOL compared with the general population or for healthy subjects. Interestingly, the score for social functioning in the SF-36 and that for the sexual and relationship domain of the IBS-QOL were somewhat higher than those of Western studies, ${ }^{34-36}$ suggesting that IBS has little impact on social function in Korean and Chinese patients. ${ }^{7,37}$ These responses may be related to the Eastern cultural milieu. Such aspects should be elaborated further by studies across the cultures. Data from nationwide cross-sectional survey in Korea ${ }^{7}$ showed functional limitations to be noted in all 8 subscales of the IBS-QOL with the mean overall score at 74.2. The lowest scores were noted on the health concern and food avoidance domains which is consistent with the West. Therefore, health was the greatest concern among IBS patients. It indicates that patients experience emotional dysphoria, ie, feeling helpless, out of control, depressed and irritable. It is noteworthy that the scores for the sexual relationship domains were 
greater than 80 , which meant that most responses were "not at all" or "slightly", indicating sexual activity was not interfered by bowel symptoms. It is contrary to the findings of the West. ${ }^{38}$ The high scores in the Sexual domain may mean that IBS marginally affects the sexual life in some Korean patients. Asians are raised and educated in a Confucian society in which both males and females avoid discussing sexual concerns.

Demographic and social factors had an effect on the HRQOL of patients with IBS. The IBS-QOL was significantly associated with female gender, symptom severity, and low level of education in Korean study. Female patients with IBS had a lower HRQOL than males, although the difference was negligible to discriminate the gender difference in HRQOL. Significant impairment of HRQOL was only observed in patients with severe symptoms, whereas patients with mild and moderate symptoms showed only mild impairment. Patients who experienced mild symptoms scored over 80 for all domains except health worry. By contrast, the overall score for IBS-QOL was less than 65 in the severe symptom group. These findings support the concept that symptom severity is an important determinant of QOL and IBS-specific QOL questionnaire has strong power to discriminate. Thus, the measurement of a patient's HRQOL could be an important element of clinical research and patient care. In a retrospective review of $\mathrm{HRQOL}$ in chronic disease, patients who were older, female, less educated, not living with a partner, and at least with 1 co-morbid condition reported the poorer level of QOL. ${ }^{39}$ Subjects with psychiatric morbidity also had a poorer QOL, thereby screening for psychiatric disorders in gastroenterology clinics is worthwhile. ${ }^{31}$

There were no differences in HRQOL between subsets of IBS, which is consistent with previous studies in which the HRQOL of subgroups of IBS was evaluated using standardized Rome criteria. ${ }^{38,40}$ Responsiveness of the IBS-QOL was validated in an open trial of tegaserod in Korean patients. ${ }^{8}$ All subscales of the IBS-QOL showed greater improvement for responders over non-responders with the greatest changes in the dysphoria, interference with activities, body image, health worry and food avoidance subscales. These findings suggest that disease-specific HRQOL could more accurately reflect the disease status of IBS and the response to treatment. The IBS-QOL questionnaire could be employed as an important outcome variable in the clinical trials of FGIDs. When looking at the subscale responses to treatments, it is clear that the psychosocial subscale scores are more responsive in explaining the nature of the improvement. For the IBS-QOL, the subscales of dysphoria, in- terference with activity and food avoidance improved more. It is perhaps not surprising that these were the scales that were more impaired prior to treatment.

\section{Conclusion}

We extensively reviewed the Asian studies that have measured HRQOL in FGIDs. We have confirmed that Asians with FGIDs showed decreased HRQOL. However, we do not have sufficient Asian data at the moment and most of them are on cross-sectional epidemiologic study. We need to know what factors would lead to patients feeling poorer QOL in Asian region. Further studies are needed to evaluate the factors influencing HRQOL, for instance, non-intestinal symptoms or psychological comorbidity. This will help not only to obtain specific patient's needs but also to provide information for making guidelines on FGIDs management.

FGIDs are common in Asia and healthcare utilization is very high. Despite this, FGIDs which are defined under Rome criteria are not being widely applied in clinical practice in Asia. Many primary care doctors do not fully comprehend the concept of functional diseases. In addition, lack of understanding FGIDs by patients might affect mental components of QOL. This could be solved through educating general practitioners as well as providing adequate information about FGIDs to general public. We know that the impact of FGIDs on population health is probably immense in Asia, however, it is still under-recognized. In light of this reality, we should place extra focus on clinical research on FGIDs.

\section{Acknowledgements}

The authors wish to thank Bo-Hyoung Jang, $\mathrm{PhD}$ for his assistance in a systematic review of the Asian literatures.

\section{References}

1. Chang L. Review article: epidemiology and quality of life in functional gastrointestinal disorders. Aliment Pharmacol Ther 2004; 20(suppl 7):31-39.

2. Constitution of the World Health Organization. In: World Health Organization. Handbook of basic documents. 5th ed. Geneva: Palais des Nations 1952:3-20.

3. Rui W, Cheng W, Ma XQ, Zhao YF, Yan XY, Jia H. Health-related quality of life in Chinese people: A population-based survey of five cities in China. Scand J Public Health 2011;39:410-418.

4. Fukuhara S, Bito S, Green J, Hsiao A, Kurokawa K. Translation, adaptation, and validation of the SF-36 Health Survey for use in 
Japan. J Clin Epidemiol 1998;51:1037-1044.

5. Azman AB, Sararaks S, Rugayah B, et al. Quality of life of the Malaysian general population: results from a postal survey using the SF-36. Med J Malaysia 2003;58:694-711.

6. Cho YK, Choi MG, Kim SH, et al. [The effect of mosapride on quality of life in functional dyspepsia.] Korean J Gastroenterol 2004;43:160-167. [Korean]

7. Park JM, Choi MG, Kim YS, et al. Quality of life of patients with irritable bowel syndrome in Korea. Qual Life Res 2009;18:435-446.

8. Kim YS, Choi SC, Park JM, et al. The effect of tegaserod on symptoms and quality of life in korean women with irritable bowel syndrome with constipation. J Neurogastroenterol Motil;16:61-70.

9. Patrick DL, Drossman DA, Frederick IO, DiCesare J, Puder KL. Quality of life in persons with irritable bowel syndrome: development and validation of a new measure. Dig Dis Sci 1998;43:400-411.

10. Talley NJ, Haque M, Wyeth JW, et al. Development of a new dyspepsia impact scale: the Nepean Dyspepsia Index. Aliment pharmacol Ther 1999;13:225-235.

11. Jones M, Talley NJ. Minimum clinically important difference for the Nepean Dyspepsia Index, a validated quality of life scale for functional dyspepsia. Am J Gastroenterol 2009;104:1483-1488.

12. Talley NJ, Verlinden M, Jones M. Validity of a new quality of life scale for functional dyspepsia: a United States multicenter trial of the Nepean Dyspepsia Index. Am J gastroenterol 1999;94:2390-2397.

13. Park JM, Choi MG, Oh JH, et al. Cross-cultural validation of Irritable Bowel Syndrome Quality of Life in Korea. Dig Dis Sci 2006;51:1478-1484.

14. Huang WW, Zhou FS, Bushnell DM, Diakite C, Yang XH. Cultural adaptation and application of the IBS-QOL in China: a disease-specific quality of life questionnaire. Research 2007;16:991-996.

15. Kanazawa M, Drossman DA, Shinozaki M, et al. Translation and validation of a Japanese version of the irritable bowel syndrome-quality of life measure (IBS-QOL-J). Biopsychosoc Med 2007;1:6.

16. Jeong JJ, Choi MG, Cho YS, et al. Chronic gastrointestinal symptoms and quality of life in the Korean population. World J Gastroenterol 2008; 14:6388-6394.

17. Choi H, Choi MG, Kim SW, et al. [The frequency of functional gastrointestinal disorders in the patients with gastrointestinal symptoms in primary care clinics and tertiary hospitals.] Korean J Gastroenterol 1999;33:741-748. [Korean]

18. Choi MG, Lee SI, Rhee JC, et al. The impact of functional dyspepsia on health related quality of life. Gastroenterology 2002;4(suppl 1):A474.

19. Chang FY, Lu CL. Irritable bowel syndrome in the 21st century: Perspectives from Asia or South-east Asia. J Gastroenterol Hepatol 2007;22:4-12.

20. Gwee KA, Bak YT, Ghoshal UC, et al. Asian consensus on irritable bowel syndrome. J Gastroenterol Hepatol 2010;25:1189-1205.

21. Lu CL, Chen CY, Lang HC, et al. Current patterns of irritable bowel syndrome in Taiwan: The Rome II questionnaire on a Chinese population. Aliment Pharmacol Ther 2003;18:1159-1169.

22. Shin $\mathrm{K}, \mathrm{Ha}$ J. [The prevalence and quality of life of nurses with irritable bowel syndrome.] Nursing Science 2006;17:1-9. [Korean]

23. Park S, Shin J. [Irritable bowel syndrome, stress, and diet, and their effects on quality of life in women college students.] Korean J Health Promot Dis Prev 2009;9:18-24. [Korean]
24. Lee S, Chang Y. [Quality of life and psychological factors in patients with irritable bowel syndrome.] Journal of Korean Living Science Research 2004;24:101-112. [Korean]

25. Hong JM. [A study on relationships among nurses's IBS, quality of life and mental health.] Nursing Science 2006;18:38-47. [Korean]

26. Lim SJ. Population-based study for prevalence of IBS, perceived stress, mental health, and health-related quality of life. Master's dissertation. Ewha Womans University, Seoul, Korea, 2008.

27. Lee $\mathrm{S}$. The prevalence of characteristics of irritable bowel syndrome in Korean population. Doctorial dissertation. Hanyang University, Seoul, Korea, 2003.

28. Gwee KA, Wee S, Wong ML, Png DJ. The prevalence, symptom characteristics, and impact of irritable bowel syndrome in an Asian urban community. Am J Gastroenterol 2004;99:924-931.

29. Kanazawa M, Endo Y, Whitehead WE, Kano M, Hongo M, Fukudo S. Patients and nonconsulters with irritable bowel syndrome reporting a parental history of bowel problems have more impaired psychological distress. Dig Dis Sci 2004;49:1046-1053.

30. Si JM, Wang LJ, Chen SJ, Sun LM, Dai N. Irritable bowel syndrome consulters in Zhejiang province: The symptoms pattern, predominant bowel habit subgroups and quality of life. World J Gastroenterol 2004;10:1059-1064.

31. Tung FY, Wu JCY, Hui AJ, Chan TSF. Psychiatric morbidity and quality of life of outpatients with irritable bowel syndrome. Hong Kong J Psychiatry 2009;19:65-71.

32. Lau EM, Chan FK, Ziea ET, Chan CS, Wu JC, Sung JJ. Epidemiology of irritable bowel syndrome in Chinese. Dig Dis Sci 2002;47:2621-2624.

33. Xiong LS, Chen MH, Chen HX, Xu AG, Wang WA, Hu PJ. A population-based epidemiologic study of irritable bowel syndrome in South China: Stratified randomized study by cluster sampling. Aliment Pharmacol Ther 2004;19:1217-1224.

34. Hahn BA, Yan S, Strassels S. Impact of irritable bowel syndrome on quality of life and resource use in the United States and United Kingdom. Digestion 1999;60:77-81.

35. Brun-Strang C, Dapoigny M, Lafuma A, Wainsten JP, Fagnani F. Irritable bowel syndrome in France: quality of life, medical management, and costs: the Encoli study. Eur J Gastroenterol Hepatol 2007;19:1097-1103.

36. Creed F, Ratcliffe J, Fernandez L, et al. Health-related quality of life and health care costs in severe, refractory irritable bowel syndrome. Ann Intern Med 2001;134:860-868.

37. Lau EM, Chan FK, Ziea ET, Chan CS, Wu JC, Sung JJ. Epidemiology ofirritable bowel syndromeinChinese. Dig Dis Sci 2002;47:2621-2624.

38. Drossman D, Morris CB, Hu Y, et al. Characterization of health related quality of life (HRQOL) for patients with functional bowel disorder (FBD) and its response to treatment. Am J Gastroenterol 2007;102:1442-1453.

39. Sprangers MA, de Regt EB, Andries F, et al. Which chronic conditions are associated with better or poorer quality of life? J Clin Epidemiol 2000;53:895-907.

40. Tillisch K, Labus JS, Naliboff BD, et al. Characterization of the alternating bowel habit subtype in patients with irritable bowel syndrome. Am J Gastroenterol 2005;100:896-904. 